\title{
Comparison of X-Ray and Gamma-Ray Dose-Response Curves for Pink Somatic Mutations in Tradescantia Clone 02*
}

\author{
A. G. Underbrink, A. M. Kellerer**, R. E. Mills, and A. H. Sparrow ${ }^{\dagger}$ \\ Department of Radiology, Radiological Research Laboratories, \\ College of Physicians and Surgeons of Columbia University, \\ New York, N.Y. 10032, USA and \\ Biology Department, Brookhaven National Laboratory, \\ Upton, N.Y. 11973, USA
}

\begin{abstract}
Summary. Microdosimetric data indicate that the mean specific energy, $\zeta$, produced by individual charged particles from $\mathrm{X}$ rays and gamma rays is different for the two radiation qualities by nearly a factor of two. In order to test whether this influences the initial, linear component in the dose-effect relations, a comparison was made between dose-response curves for pink somatic mutations in Tradescantia clone 02 stamen hairs following $\mathrm{X}$ and gamma irradiations. Absorbed doses ranged from 2.66 to $300 \mathrm{rad}$. The results are in agreement with predictions made on the basis of microdosimetric data. At low doses gamma rays are substantially less effective than $X$ rays. The $R B E$ of gamma rays vs. $X$ rays at low doses was approximately 0.6 , a value lower than those usually reported in other experimental systems.
\end{abstract}

\section{Introduction}

Previous extensive experiments with monoenergetic neutrons and with $\mathrm{X}$ rays have led to the conclusion that the induction of pink somatic mutations in stamen hairs of Tradescantia clone 02 follows a linear-quadratic relation in absorbed dose at low and intermediate dose levels [1-4]. The linear component in the dose-effect relation represents the intra-track effect, i.e., it results from the synergism of lesions produced by the same particle track. The quadratic component represents the intertrack effect, i.e., it results from the synergism of lesions produced by separate particle tracks. The magnitude of the linear component is, therefore, dependent on the mean energy concentrations produced by individual particle tracks. The quantitative

* Research supported by Public Health Service Research Grant No. CA-12536 from the National Cancer Institute and the U.S. Energy Research and Development Administration at Brookhaven National Laboratory. By acceptance of the article, the publisher and/or recipient acknowledges the U.S. Government's right to retain a nonexclusive, royalty-free license in and to any copyright concerning this paper

** Present address: Institut für Med. Strahlenkunde der Universität, Versbacher Landstr. 5, D-8700 Würzburg, Federal Republic of Germany 
analysis shows [5] that the ratio of the coefficients in the linear and the quadratic terms is equal to the dose average of specific energy, $\zeta$, produced by individual charged particles in the sensitive site. This term is large for neutrons, and accordingly the linear term predominates. For $\mathrm{X}$ rays, however, the linear term is of consequence only at low doses. It is, therefore, in most experimental systems difficult to assess the magnitude of this linear term accurately.

The induction of pink somatic mutations in stamen hairs of Tradescantia is an experimental system particularly useful for the study of effects of low doses of ionizing radiations. The reason is that extremely large numbers of exposed cells can be examined. Accordingly, it has been possible in earlier studies [1-4] to determine not only the dose-effect relations for neutrons but to obtain also accurate results concerning the initial linear component of the dose-effect relation for $\mathrm{X}$ rays [1, 2]. The present study goes one step further. It is not concerned with the comparison of two types of radiations which are very different in quality such as $X$ rays and neutrons; instead $X$ rays are compared with gamma rays, i.e., two very similar radiation qualities are used.

Microdosimetric data indicate, that despite the similarity of the two radiation qualities, there may be a substantial difference in effectiveness at low doses. The quantity, $\zeta$, is about twice as large for $\mathrm{X}$ rays as for gamma rays [5]. If the microdosimetric explanation applies, one would therefore expect that the initial linear component is about twice as large for $\mathrm{X}$ rays than for gamma rays. On the other hand, if the linear components are found to be similar for the two radiation qualities, one would have to conclude that the microdosimetric interpretation of the linear term as intra-track effect does not apply i.e., that the initial linear term in absorbed dose for sparsely ionizing radiation is not due to the occasional occurrence of high energy concentrations in the site. Instead one would have to assume that the linear term reflects a mechanism which is inherently proportional to specific energy and not to the square of specific energy.

There is also an additional reason for this experimental investigation. In previous dose-rate studies on the induction of these mutations [6], it was found to be necessary in some cases to substitute gamma rays for $\mathrm{X}$ rays. A preliminary study indicated that the $\mathrm{RBE}$ of ${ }^{137} \mathrm{Cs}$ gamma rays relative to $250 \mathrm{kVp} \mathrm{X}$ rays was about 0.82 for pink somatic mutations [6]. Since these dose-rate experiments are currently being expanded, it was desirable to do a more comprehensive determination of RBE between these two radiation qualities at intermediate to high doses (over $10 \mathrm{rad}$ and up to about $300 \mathrm{rad}$ ). Such a study should provide more accurate conversion factors for these experiments.

\section{Materials and Methods}

Young unrooted cuttings of Tradescantia clone 02 were used for all experiments. The procedures for growing and handling of the plants before and after irradiation have been described previously $[1,3,6,7]$. The stamen hairs were scored for pink mutant events, and values were averaged from total mutant events divided by total stamen hairs scored from days 11 through 15 after irradiation $[1,7]$.

Data were analyzed at Brookhaven National Laboratory by computer programs composed by Mr. K. H. Thompson. 


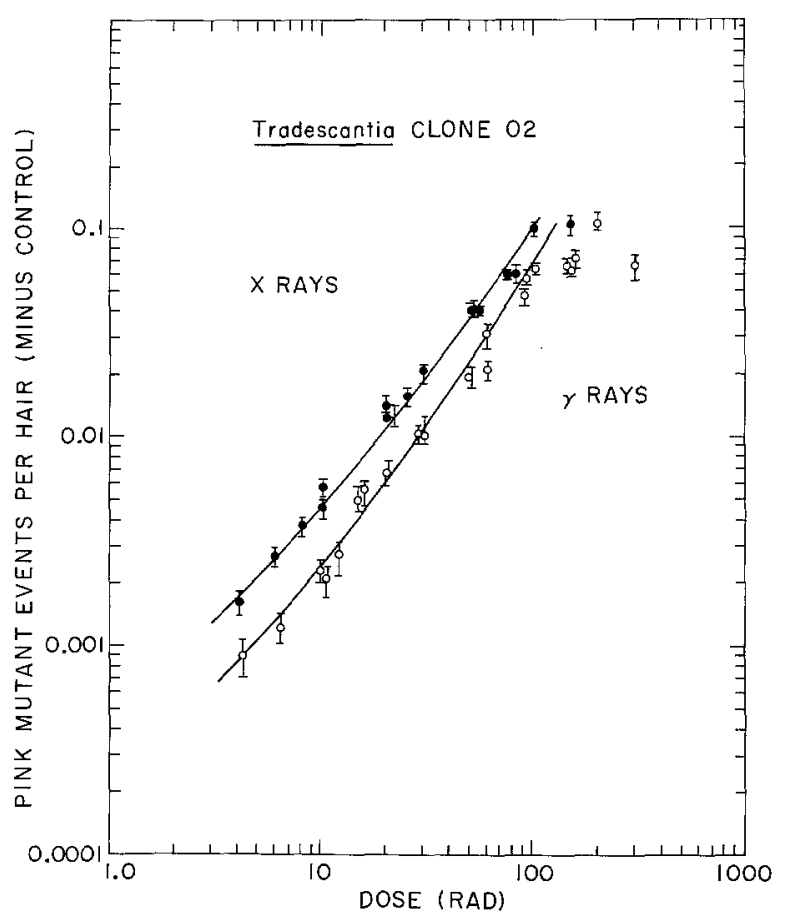

Fig. 1. Plot of pink mutant events per hair (minus control) vs. X-and gamma-ray dose. The solid lines correspond to Equations (1) and (2)

$X$ Irradiations. $\mathrm{X}$ irradiations were performed concurrently with the ${ }^{137} \mathrm{Cs}$ gamma irradiations in four separate sets of experiments (Table 1). For the first series of $\mathrm{X}$ irradiations at doses of 2.66-6.0 rad (exp. set no. 1, Table 1), the machine was operated at $250-\mathrm{kVp}$ and $12 \mathrm{~mA}$ with $1.5 \mathrm{~mm} \mathrm{Cu}+1 \mathrm{~mm} \mathrm{Al}$ filtration. Target to Xray tube distance was $126 \mathrm{~cm}$. The resulting absorbed dose rate was $9.6 \mathrm{rad} / \mathrm{min}$. The low dose rate was chosen in these experiments to keep the conditions similar to those used in the gamma irradiations. At the Controlled Environment Radiation Facility (CERF), Brookhaven, it was not feasible to deliver the two lowest gamma exposures at $30 \mathrm{rad} / \mathrm{min}$.

Dosimetry was performed with a Landsverk chamber. Both the dosimetry and irradiations were done atop 8 fiber boards on a rotating table with the cuttings covered with $1.6 \mathrm{~mm}$ lucite.

The $\mathrm{X}$ irradiations for the other three sets of experiments (exp. sets no. 2-4, Table 1) were done in identical fashion except that target to X-ray tube distance was reduced to $69.5 \mathrm{~cm}$, and the dose rate was increased to $30 \mathrm{rad} / \mathrm{min}$. From previous experience with this test system, we did not expect dose-rate effects at doses below about $10 \mathrm{rad}[1,6]$. The experiments (Table 1, Fig. 1) involving doses around $10 \mathrm{rad}$ of either radiation support this assumption.

Gamma Irradiations. The $12,000 \mathrm{Ci}^{137} \mathrm{Cs}$ source at CERF was used for the gamma exposures. The cuttings were taped to upright sheets of $1.6 \mathrm{~mm}$ lucite and 
placed in arcs in front of the source. The absorbed dose rate for the first set of experiments (exp. set no. 1, Table 1) was $10.9 \mathrm{rad} / \mathrm{min}$ and $30 \mathrm{rad} / \mathrm{min}$ for the other three sets. Dosimetry was performed with both high energy calibrated Victoreen condenser type $\mathbf{R}$ chambers and a tissue-equivalent ionization chamber. Measurements made with the former yield exposures directly, and exposure rates were determined by timing the irradiations. Exposures were converted to absorbed dose in rad by the conversion factor 0.957 .

Measurement of ionization current in the tissue-equivalent chamber was accomplished by means of a custom built portable electrometer system. Current was converted to measurable voltage by an FET electrometer with a feedback resistance of $10^{10} \Omega$, and this was converted in turn to a train of pulses having a proportional frequency. Determination of this frequency, therefore, gives exposure rate; and total scaler count gives exposure. There is no upper limit to the exposure which can be monitored in this manner.

The calibration factor used to convert counts to exposure was determined from measurements of a $10 \mathrm{Ci}^{137} \mathrm{Cs}$ source whose output was well known. Measurements of exposures at CERF using both techniques were generally in good agreement (within 2\%).

\section{Results and Discussion}

Data collected from the four sets of experiments are given in Table 1. Pink mutant events per hair (minus control) are plotted against dose in Figure 1. One data point, namely the one for $2.66 \mathrm{rad}$ of $\mathrm{X}$ rays, has not been included in Figure 1. The reason is that in this case an anomalous variation of mutation rate occurred over the five day scoring period from days 11 through 15 after irradiation. On days 14 and 15 the mutation rates were an order of magnitude smaller than the rates observed on day 13. We have found no explanation for this anomaly and have, therefore, omitted this point. It is, however, included in Table 1 and is there put between brackets.

Previous experimental data and theoretical considerations concerning $\mathrm{X}$ ray dose-response curves in this system [1] suggest that the ascending portion of the $\mathrm{X}$ ray curve is the sum of a linear and quadratic term in absorbed dose. At low doses the data are consistent with a slope $=+1$; at higher doses the curve steepens.

The curves in Figure 1 result from a visual fit of the linear-quadratic relation to the data. The two curves correspond to the equation:

$$
y(D)=4.23 \times 10^{-4} D+6.5 \times 10^{-6} D^{2}
$$

for $\mathrm{X}$ rays, and to the equation:

$$
y(D)=1.98 \times 10^{-4} D+5.2 \times 10^{-6} D^{2}
$$

for gamma rays. The controls are subtracted in both situations; $y(D)$ is the frequency of pink mutations per hair, and $D$ is the absorbed dose in rad. The data are less complete than the data which in an earlier analysis [8] have been subjected to a rigorous statistical evaluation. For this reason, and also because the data result from different experiments which have not all been performed simultaneously, no confi- 
dence intervals are given for the coefficients in the linear and the quadratic terms in the absorbed dose. Furthermore, no attempt has been made to fit the data in the range of high doses where the incidence goes through a plateau and then declines. The present data are not sufficient to permit a quantitative assessment of the functional dependence of the effect on absorbed dose in this dose range. Due to these limitations in the statistical treatment the following theoretical statements must be considered as tentative. Together with similar results obtained by Schmid et al. [13] in a different experimental system, namely chromosome aberrations in human lymphocytes, they may nevertheless throw some light on the still unresolved question of the relative biological effectiveness of gamma rays vs. $X$ rays.

According to the microdosimetric analysis, the linear-quadratic dose-effect relation results from the interaction of pairs of sublesions within sensitive sites of the nuclei of the cell. In the present experiments it may be assumed that the sublesions are chromosome breaks, and that the effect results from chromosome aberrations which are due to the interaction of two chromosome breaks. The microdosimetric interpretation is, therefore, closely related to earlier ideas advanced, for example, by Lea [9].

The linear-quadratic dose-effect relation can be written in the form:

$$
y(D)=k\left(\zeta D+D^{2}\right)
$$

where $\zeta$ is the mean specific energy produced by individual charged particles in a sensitive site, and $k$ is proportional to the efficiency of the production of sublesions and also depends on the interaction probability of sublesions [5]. With this modification one obtains the relation:

$$
y(D)=6.5 \times 10^{-6}\left(65 D+D^{2}\right)
$$

for $\mathrm{X}$ rays, and the relation:

$$
y(D)=5.2 \times 10^{-6}\left(38 D+D^{2}\right)
$$

for gamma rays. The absorbed dose, $D$, is again expressed in rad.

The values of the coefficient $k$ for the two types of radiations are similar. A slightly larger value of $k$ is obtained for the more densely ionizing $\mathrm{X}$ rays, but the difference does not appear to be statistically significant.

The values of $\zeta$ obtained for gamma rays and $X$ rays are $65 \mathrm{rad}$ and $38 \mathrm{rad}$, i.e., the value $\zeta$ for gamma rays is smaller by a factor of 0.58 than the value for $X$ rays. This is in agreement with the predictions made on the basis of microdosimetric data. The observed values correspond to a site diameter of approximately $1.3 \mu \mathrm{m}$. An earlier microdosimetric calculation [10] indicates that the values of $\zeta$ for ${ }^{60} \mathrm{Co}$ gamma rays and $200-\mathrm{kVp} X$ rays differ by about a factor of 2 . More recent experimental investigations by Ellett and Braby [11] for $250-\mathrm{kVp} X$ rays compared with results obtained by $\mathrm{R}$. Dvorak [15] for ${ }^{137} \mathrm{Cs}$ gamma rays also indicate a difference by a factor of about 2 . The results obtained in the present experiments exhibit a ratio which is not quite as large as 2 but is close to it. The results, therefore, confirm that at low doses gamma rays are substantially less effective than $\mathrm{X}$ rays due to the more infrequent occurrence of high energy concentrations in the sensitive sites of the cell. 


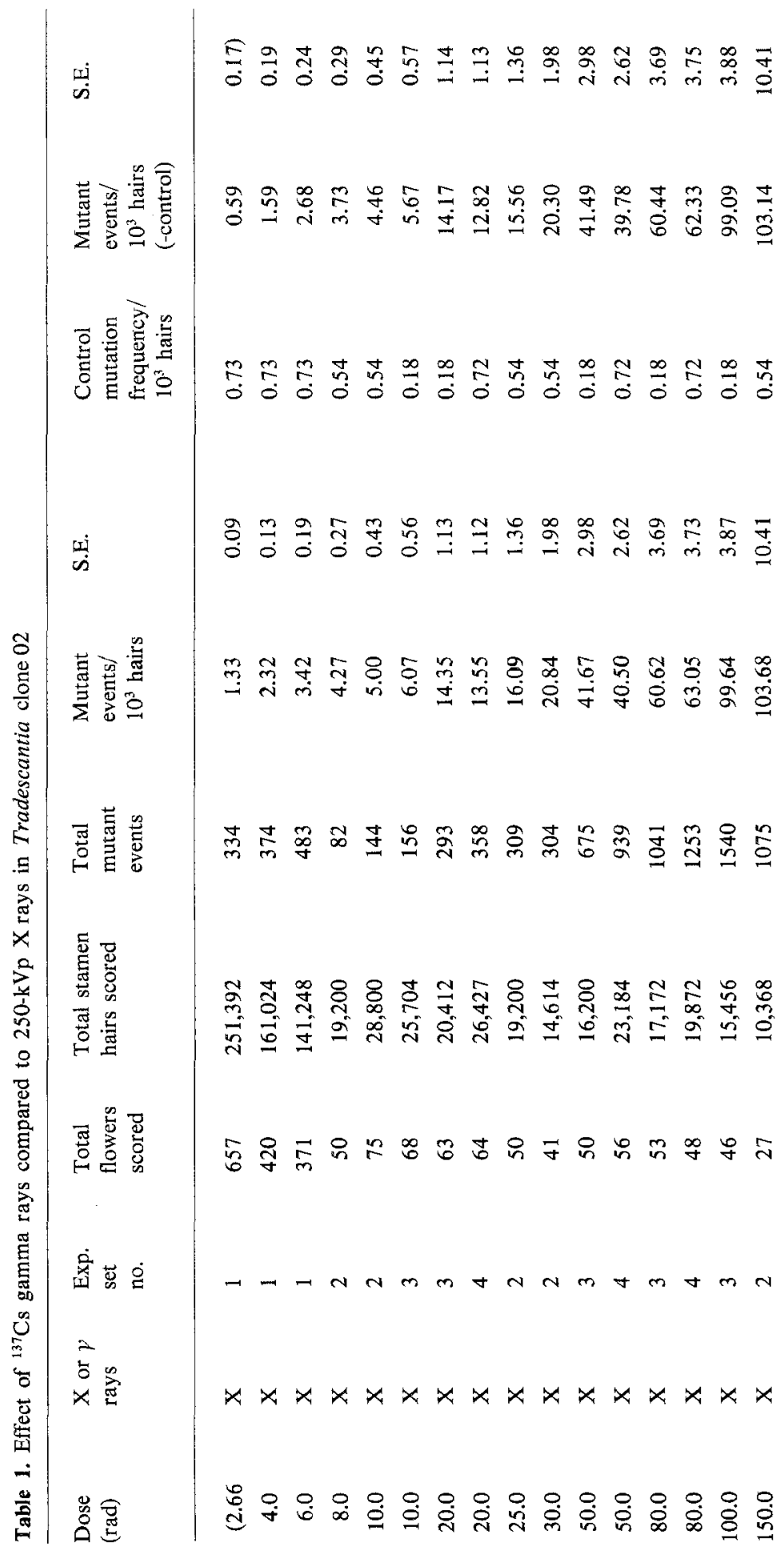




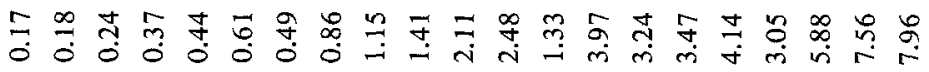

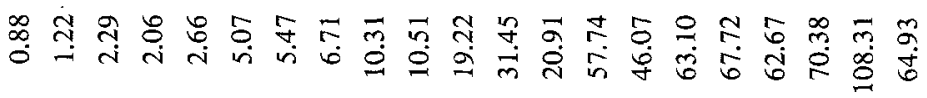

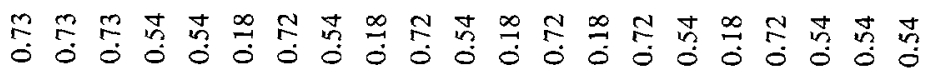

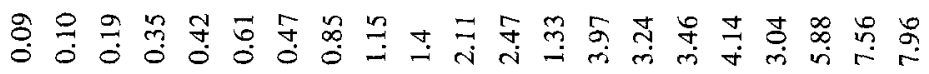

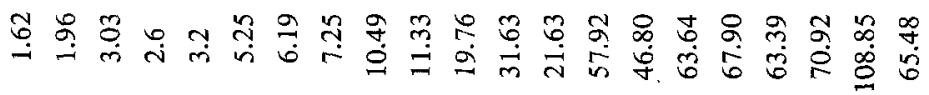

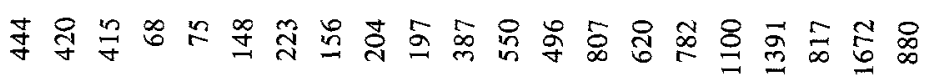

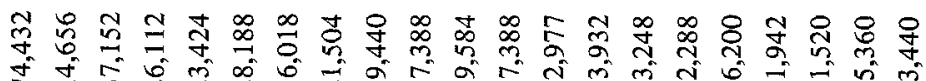

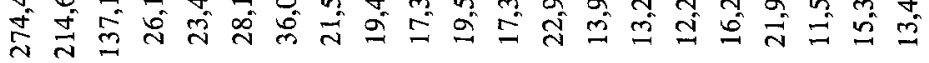

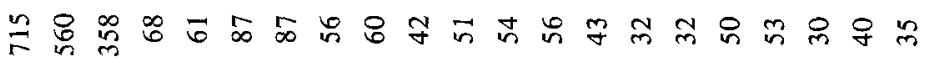

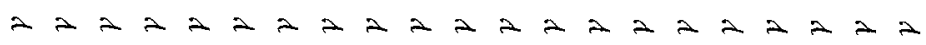

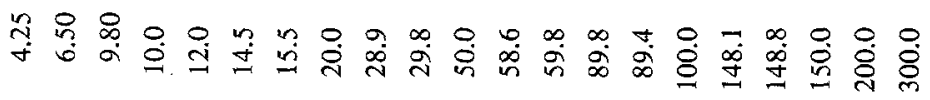


With increasing absorbed doses the role of the quadratic component in the doseeffect relation becomes more important and consequently the difference in biological effectiveness of the two radiations is less expressed.

In earlier experiments on the induction of pink mutations in Tradescantia clone $02[1,8]$, a dose-effect relation for $250-\mathrm{kVp} \mathrm{X}$ rays has been obtained which has a linear component nearly equal to the one in the present experiments while its quadratic component is substantially larger than in the present experiment. The earlier results [8] were represented by the equation ( $D$ expressed in rad):

$$
y(D)=4.0 \times 10^{-4} D+3.1 \times 10^{-5} D^{2}
$$

Comparison with Equation 1 shows a striking difference in the magnitude of the quadratic component. We cannot at present identify the factors responsible for this change of the dose-effect relation in separate experiments. However, the observation strongly underlines the necessity that the study of dose modifying factors such as RBE should as far as possible be based on experiments performed simultaneously.

The finding of a value of approximately 0.6 for the RBE of gamma rays vs. $\mathrm{X}$ rays at low doses may appear to contradict the usual assumption (see for example Sinclair et al., [12] ) of a value of about 0.85 for the RBE of gamma rays vs. X rays. However, there are two reasons which may account for this difference. The first reason is that the value normally quoted refers to cell lethality, while our results pertain to a more specific and nonlethal cell damage. In earlier experiments on the induction of chromosome aberrations in human lymphocytes by fast electrons and $\mathrm{X}$ rays, Schmid et al., [13] have come to conclusions which are very similar to our present results.

A second reason for the difference between our observations and the commonly accepted ratio of the effectiveness of gamma rays vs. X rays may be that we are dealing with very low doses, while studies of RBE for cell lethality such as those recently reported by Malone et al. [14] for mammalian cells are mostly performed at higher doses. In experiments concerning cell lethality it is usually not possible to obtain accurate survival data at absorbed doses of sparsely ionizing radiations significantly below $100 \mathrm{rad}$. According to the linear-quadratic dose-effect relation, the $\mathrm{RBE}$ of gamma rays should indeed come close to unity as the doses are increased toward values where the quadratic component predominates. However, it will be desirable to obtain more accurate data both in our system and in the studies of cell lethality.

Acknowledgements. The authors thank Mrs. Diane Sautkulis $\dagger$ for technical assistance and Miss Virginia Pond for suggestions concerning the manuscript.

\section{References}

1. Sparrow, A. H., Underbrink, A. G., Rossi, H. H.: Mutations induced in Tradescantia by small doses of X rays and neutrons: analysis of dose-response curves. Science 176, 916-918 (1972)

2. Underbrink, A. G., Sparrow, A. H.: The influence of experimental end points, dose, dose rate, neutron energy, nitrogen ions, hypoxia, chromosome volume and ploidy level on RBE in Tradescantia stamen hairs and pollen. In: Biological Effects of Neutron Irradiation, pp. 185-213. Vienna: IAEA 1974 
3. Underbrink, A. G., Sparrow, R. C., Sparrow, A. H., Rossi, H. H.: Relative biological effectiveness of $\mathrm{X}$ rays and $0.43-\mathrm{MeV}$ monoenergetic neutrons on somatic mutations and loss of reproductive integrity in Tradescantia stamen hairs. Radiat. Res. 44, 187-203 (1970)

4. Underbrink, A. G., Sparrow, A. H., Sautkulis, D., Mills, R. E.: Oxygen enhancement ratios (OERs) for somatic mutations in Tradescantia stamen hairs. Radiat. Bot. 15, 161-168 (1975)

5. Kellerer, A. M., Rossi, H. H.: The theory of dual radiation action. Curr. Top. Radiat. Res. Quart. 8, 85-158 (1972)

6. Nauman, C. H., Underbrink, A. G., Sparrow, A. H.: Influence of radiation dose rate on somatic mutation induction in Tradescantia stamen hairs. Radiat. Res. 62, 79-96 (1975)

7. Underbrink, A. G., Schairer, L. A., Sparrow, A. H.: Tradescantia stamen hairs: a radiobiological test system applicable to chemical mutagenesis. In: Chemical Mutagens: Principles and Methods for Their Detection, Vol. 3, pp. 171-207 (A. Hollaender, Ed.) New York: Plenum Press 1973

8. Kellerer, A. M., Brenot, J.: On the statistical evaluation of dose-response functions. Rad. Environm. Biophys. 11, 1-13 (1974)

9. Lea, D. E.: Action of Radiations on Living Cells. Cambridge, England: Cambridge University Press 1974

10. Hug, O., Kellerer, A. M.: Stochastik der Strahlenwirkung. Berlin-Heidelberg-New York: Springer 1966

11. Ellett, W. H., Braby, L. A.: The microdosimetry of $250-\mathrm{kVp}$ and $65-\mathrm{kVp} \mathrm{X}$ rays, Co-gamma rays, and tritium beta particles. Radiat. Res. 51, 229-243 (1972)

12. Sinclair, W. K., Gunter, S. E., Cole, A.: The relative biological effectiveness of $200-\mathrm{kVp}$ X rays, cobalt-60 gamma-rays, and 22-MeVp X-rays, determined from the dose-survival curve of Saccharomyces cerevisiae. Radiat. Res. 10, 418-432 (1959)

13. Schmid, E., Rimpl, G., Bauchinger, M.: Dose-response relation of chromosome aberrations in human lymphocytes after in vitro irradiation with $3 \mathrm{MeV}$ electrons. Radiat. Res. 57, 228-238 (1973)

14. Malone, J. F., Porter, D., Hendry, J. H.: The r.b.e. of ${ }^{60} \mathrm{Co}$ gamma rays with respect to $300 \mathrm{kVp}$ Xrays for the survival of HeLa S-3 and CHO cells, irradiated in different states of proliferation. Int. J. Radiat. Biol. 26, 355-362 (1974)

15. Dvorak, R. F.: Event distributions for monoenergetic photons. In: Annual Report on Research Project, Rad. Res. Lab. Columbia University, pp. 45-56, USAEC - COO-3243-4 (1975) 